\title{
Modulation of long-term potentiation-like cortical plasticity in the healthy brain with low frequency-pulsed electromagnetic fields
}

Enrico Premi ${ }^{1,2^{*}} \mathbb{D}$, Alberto Benussi ${ }^{2}$, Antonio La Gatta ${ }^{3}$, Stefano Visconti ${ }^{4}$, Angelo Costa ${ }^{1}$, Nicola Gilberti ${ }^{1}$, Valentina Cantoni ${ }^{2}$, Alessandro Padovani ${ }^{2}$, Barbara Borroni ${ }^{2}$ and Mauro Magoni ${ }^{1}$

\begin{abstract}
Background: Non-depolarizing magnetic fields, like low frequency-pulsed electromagnetic fields (LF-PEMFs) have shown the ability to modulate living structures, principally by influencing synaptic activity and ion channels on cellular membranes. Recently, the CTU Mega 20 device was presented as a molecular accelerator, using energy up to $200 \mathrm{~J}$ and providing high-power (2 Tesla) pulsating fields with a water-repulsive (diamagnetic) action and tissue biostimulation. We tested the hypothesis that LF-PEMFs could modulate long-term corticospinal excitability in healthy brains by applying CTU Mega $20^{\circledR}$. Ten healthy subjects without known neurological and/or psychiatric diseases entered the study. A randomized double-blind sham-controlled crossover design was employed, recording TMS parameters (amplitude variation of the motor evoked potential as index of cortical excitability perturbations of the motor system) before (pre) and after (post + 0, + 15, + 30 min) a single CTU Mega 20 session on the corresponding primary righthand motor area, using a real (magnetic field $=2$ Tesla; intensity $=90 \mathrm{~J}$; impulse frequency $=7 \mathrm{~Hz}$; duration $=15 \mathrm{~min}$ ) or sham device. A two-way repeated measures ANOVA with TIME (pre, post + 0, + 15, + 30 min) and TREATMENT (real vs. sham stimulation) as within-subjects factor was applied.

Results: A significant TIME $\times$ TREATMENT interaction was found $(p<0.001)$. Post hoc comparisons showed a significant effect of TIME, with significant differences at $+0,+15$ and +30 min compared to baseline after real stimulation (all $p<0.05$ ) but not after sham stimulation (all $p<0.05$ ) and significant effects of TREATMENT, with significant differences at $+0,+15$ and +30 min for real stimulation compared to sham stimulation (all $p<0.005$ ). No significant depolarizing effects were detected throughout the (real) stimulation.
\end{abstract}

Conclusions: Our proof-of-concept study in healthy subjects supports the idea that non-ionizing LF-PEMFs induced by the CTU Mega 20 diamagnetic acceleration system could represent a new approach for brain neuromodulation. Further studies to optimize protocol parameters for different neurological and psychiatric conditions are warranted.

Trial Registration The present work has been retrospectively registered as clinical trial on ClinicalTrials.gov NCT03537469 and publicly released on May 24, 2018

Keywords: Long-term potentiation-like cortical plasticity, Low frequency-pulsed electromagnetic fields, Diamagnetism, Neuroplasticity

\footnotetext{
*Correspondence: zedtower@gmail.com

1 Stroke Unit, Azienda Socio Sanitaria Territoriale "Spedali Civili", "Spedali

Civili" Hospital, Piazza Spedali Civili 1, 25123 Brescia, Italy

Full list of author information is available at the end of the article
}

c The Author(s) 2018. This article is distributed under the terms of the Creative Commons Attribution 4.0 International License (http://creativecommons.org/licenses/by/4.0/), which permits unrestricted use, distribution, and reproduction in any medium, provided you give appropriate credit to the original author(s) and the source, provide a link to the Creative Commons license, and indicate if changes were made. The Creative Commons Public Domain Dedication waiver (http://creativecommons.org/ publicdomain/zero/1.0/) applies to the data made available in this article, unless otherwise stated. 


\section{Background}

Several studies have investigated the effects of different stimulation methods in modulating human brain plasticity [1-3]. Among these, the paired associative stimulation (PAS) paradigms were shown to modulate the excitability of corticospinal fibers related to the primary motor cortex, as a form of long-term modulation, including long-term potentiation (LTP) or depression (LTD) linked to synaptic plasticity $[4,5]$. All these approaches induce electric currents to obtain a depolarization in the stimulated brain regions $[6,7]$. However, even non-depolarizing magnetic fields, as in static magnets [8-10] or Low Frequency-Pulsed Electromagnetic Fields (LF-PEMFs) [11, 12], have shown the potential to modulate living structures [8, 13]. In particular, these non-depolarizing approaches seem to influence synaptic activity and ion channels on cellular membranes [8]. Indeed, it has been suggested that LF-PEMF can influence numerous types of changes in cells including migration, cell differentiation, stress response, potentially affecting morphology, migration of embryonic cells, and cell reprogramming [13-17]. Furthermore, it has been reported that LF-PEMF promotes osteogenic and neurogenic differentiation, which has been clinically used to repair bone fractures, promote wound healing $[18,19]$, and has been shown to have a neuroprotective effect after ischemic stroke in mice during the recovery process [11]. Several trials have also assessed the effects of LF-PEMF on major depressive disorder and unipolar or bipolar depression [20-22]. Moreover, LF-PEMF has been reported to influence brain glucose metabolism, thus affecting local brain activity [23]. Collectively, these studies indicate that LF-PEMF may be involved in neuroprotection.

LF-PEMFs $(<50 \mathrm{~Hz})$ can be considered as a class of non-ionizing radiation with an associate energy $<12$ electronvolt $(\mathrm{eV})$, not enough to induce ionization phenomena [24], but with potential effects on biological components [25]. Recently, the CTU Mega $20^{\circledR}$ device (see Fig. 1) was presented as a molecular accelerator, using an energy up to $200 \mathrm{~J}$ and providing high-power (2 Tesla) pulsating fields with a water-repulsive (diamagnetic) action with a consequent tissue biostimulation [25, 26]. In this research we tested the hypothesis that LFPEMFs could modulate long-term corticospinal excitability in the healthy brain by applying transcranial pulsed magnetic fields with CTU Mega $20^{\circledR}$ (http://www.peris o.ch/). Therefore, we employed single-pulse transcranial magnetic stimulation (TMS), which allows an in vivo registration of the amplitude variation of the motor evoked potentials as a tool to explore cortical excitability perturbations of the motor system after CTU Mega 20 application.

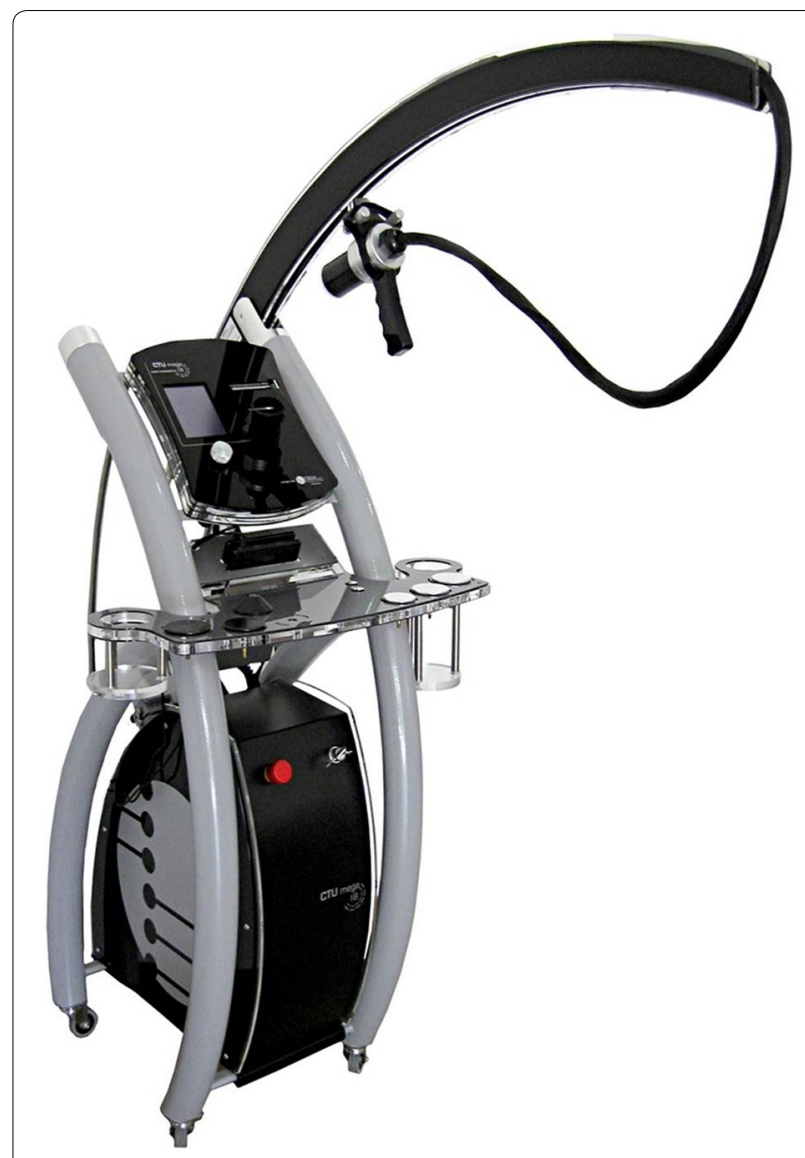

Fig. 1 CTU Mega 20 device. The original equipment used in the study, directly provided by PERISO SA (http://www.periso.ch/)

\section{Methods \\ Subjects}

Ten healthy subjects without known neurological or psychiatric diseases were recruited for this study (mean age \pm standard deviation: $25.5 \pm 3.8$ years) (see Table 1 ).

The subjects and operators who performed TMS were blinded to the type of stimulation applied.

Written informed consent was obtained from all subjects according to the Declaration of Helsinki. The study protocol was approved by the local ethics committee. This study adheres to CONSORT guidelines (http://www. consort-statement.org/) (see Additional file 1 for CONSORT checklist).

\section{CTU Mega 20 stimulation}

The CTU Mega 20 diamagnetic acceleration system discharges high-field magnetic impulses (with a duration of $5 \mathrm{~ms}$ and a period of $1000 \mathrm{~ms}$ ), generating a magnetic field up to 2 Tesla, with a frequency of $7500 \mathrm{~Hz}$ in a volume of approximately $27 \mathrm{~cm}^{3}$ [12]. See Additional file 2 
Table 1 Demographic characteristics and neurophysiological parameters

\begin{tabular}{|c|c|c|c|}
\hline Variable & Real $(n=10)$ & Sham $(n=10)$ & $p$ \\
\hline Age $($ mean $\pm S D)$ & $25.5 \pm 3.8$ & $25.5 \pm 3.8$ & - \\
\hline Gender \% (no female) & $50(5)$ & $50(5)$ & - \\
\hline Educational level, years & $21.5 \pm 1.9$ & $21.5 \pm 1.9$ & - \\
\hline Handedness \% (no right) & $100(10)$ & $100(10)$ & - \\
\hline Correct hypothesis on treatment \% & 50 & 50 & - \\
\hline rMT (\% of MSO) & $46.4 \pm 6.5$ & $46.2 \pm 6.5$ & n.s. \\
\hline Corticospinal excitability baseline (mV) & $1.01 \pm 0.12$ & $1.05 \pm 0.05$ & n.s. \\
\hline Corticospinal excitability post + 0 (mV) & $1.60 \pm 0.19$ & $1.10 \pm 0.14$ & $p=0.001$ \\
\hline Corticospinal excitability post + 15 (mV) & $1.69 \pm 0.18$ & $1.12 \pm 0.21$ & $p<0.001$ \\
\hline Corticospinal excitability, post $+30(\mathrm{mV})$ & $1.57 \pm 0.34$ & $1.07 \pm 0.20$ & $p=0.002$ \\
\hline
\end{tabular}

MSO max stimulator output, SD standard deviation, $m V$ millivolt

for a detailed technical description of CTU Mega 20 physics principles and function.

To assess the effect of LF-PEMFs provided by CTU Mega 20 we employed a randomized double-blind shamcontrolled crossover design, recording TMS parameters before (pre) and after (post $+0,+15,+30 \mathrm{~min}$ ) a singlesession of CTU Mega 20 on the corresponding primary right-hand motor area, using a real (magnetic field $=2$ Tesla; intensity $=90 \mathrm{~J}$; frequency of impulses $=7 \mathrm{~Hz}$; duration $=15 \mathrm{~min}$ ) or the sham device. Subjects were randomly assessed for real or sham protocol stimulation, in a 1:1 ratio, with a mean interval of $16.9 \pm 2.1$ days between sessions.

To detect differences in the perception of the stimulation, we asked the patients whether they thought they were receiving real or sham stimulation at the end of each treatment.

\section{Transcranial magnetic stimulation}

TMS was performed with a figure-eight coil (loop diameter $70 \mathrm{~mm}$ ) connected to a Magstim $200^{2}$ stimulator (Magstim Company, Oxford, UK). The magnetic stimuli had a monophasic current waveform (rise time of $100 \mu \mathrm{s}$, decaying back to zero over $800 \mu \mathrm{s})$. The motor evoked potentials (MEPs) were registered from the right first dorsal interosseous muscle (FDI) through surface $\mathrm{Ag} / \mathrm{AgCl}$ electrodes placed in a belly-tendon montage and acquired using a Biopac MP-150 electromyograph (BIOPAC Systems Inc., Santa Barbara, CA, USA), as previously reported [27].

The TMS coil was held tangentially over the scalp zone related to the primary hand motor area contralateral to the target muscle, with the coil handle pointed $45^{\circ}$ posteriorly and laterally to the sagittal plane. The motor region was considered as the location where TMS consistently produced the largest MEP size at $120 \%$ of the resting motor threshold (rMT) in the target muscle. The region was marked with a felt tip pen on the scalp to guarantee constant placement of the coil during the whole experiment. The stimulator intensity was set to evoke a MEP approximately $1 \mathrm{mV}$ peak-to-peak in the relaxed FDI at baseline, and was kept constant during the whole session. MEP amplitude measurements (average of 25 responses) were performed at baseline and at 0,15 , and $30 \mathrm{~min}$ after sham or real stimulation. The inter trial interval was set at $5 \mathrm{~s}( \pm 10 \%)$.

Throughout the experiment, complete muscle relaxation was guaranteed by audio-visual feedback where appropriate. Trials were discarded if EMG activity exceeded $100 \mu \mathrm{V}$ in the $250 \mathrm{~ms}$ prior to TMS stimulus delivery. All participants were able to understand instructions, obtaining a full muscle relaxation.

\section{Statistical analysis}

Neurophysiological parameters were compared by means of two-way repeated measures ANOVA with TIME (pre, post $+0,+15,+30 \mathrm{~min}$ ) and TREATMENT (real vs. sham stimulation) as within-subjects factor. When a significant main effect was reached, post hoc tests with Bonferroni correction for multiple comparisons were conducted to analyze group-differences at respective interstimulus intervals s or time points. Mauchly's test was used to test for assumption of sphericity, while the Greenhouse-Geisser epsilon determination was used to correct in case of sphericity violation.

Spearman's rank-order correlation was used to assess the association between the percentage of increase in MEP amplitude and baseline rMT.

Statistical significance was assumed at $p<0.05$. Data analyses were carried out using SPSS 21.0 software.

\section{Results}

Regarding the differences in the subjects' perception of the stimulation, there was no statistically significant association between the type of stimulation and its 
perception, as assessed by Fisher's exact test, $p=1.00$, suggesting that real stimulation could not be distinguished from sham stimulation.

Two-way repeated measures ANOVA performed on corticospinal excitability revealed a significant TIME $\times$ TREATMENT interaction, $F(3,27)=0.453$, $p<0.001$, partial $\eta^{2}=0.645$. A significant main effect of TIME was observed, with significant differences in post hoc tests at $+0,+15$ and +30 min compared to baseline after real stimulation (all $p<0.05$ ) but not after sham stimulation (all $p>0.05$ ). There was also a significant main effect of TREATMENT, with significant differences at $+0,+15$ and +30 min versus baseline, for real stimulation but not for sham stimulation (all $p<0.005$ ) (see Table 1, Fig. 2).

There was no significant association between the percentage of increase in average MEP amplitude and baseline rMT in both groups (real stimulation: $\mathrm{r}_{\mathrm{s}}=0.10$, $p=0.776$; sham stimulation: $\left.\mathrm{r}_{\mathrm{s}}=-0.46, p=0.177\right)$.

During the treatment phase (application of real or sham CTU Mega 20 protocol), EMG activity at highgain amplification was monitored to highlight possible depolarizing effects, which were however absent throughout the stimulation.

\section{Discussion}

In this study, we employed a randomized double-blind sham-controlled crossover design (to control for known and unknown factors that could potentially influenced brain activity and TMS registration) to demonstrate that Low Frequency-Pulsed Electromagnetic Fields (LFPEMFs) induced by CTU Mega 20 were able to modulate cortical excitability in human brains, even after a singleshot application. As described above, these findings were not influenced by subject treatment expectation (real stimulation versus sham), considering its potential effect on brain activity and consequently on TMS parameters [28]. In line with transcranial static magnetic field stimulation [10, 23, 29], cortical excitability enhancement was not directly related to induced electric currents, as is the case for other neuromodulation TMS-based techniques.

In our experiment, by providing 15-min pulsed-magnetic stimulus on the primary motor area, we obtained a persistent increase of more than $60 \%$ in corticospinal excitability (as an index of Long-Term Potentiation-Like Cortical Plasticity), recording the MEP from the contralateral first dorsal interosseous muscle. This perturbation lasted for at least for 30 min after the stimulation protocol, potentially maintaining a significant difference

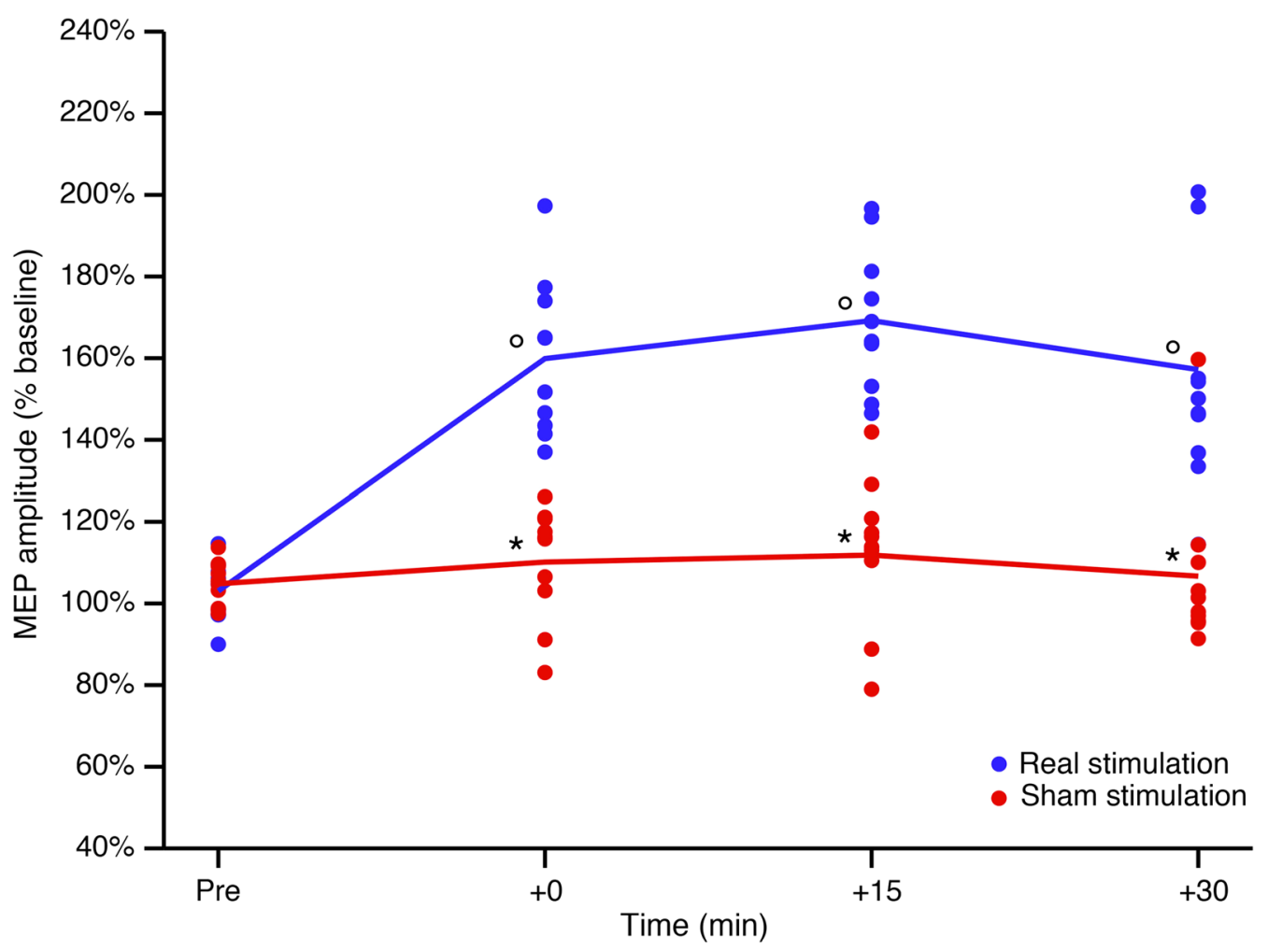

Fig. 2 Corticospinal excitability after real and sham stimulation. Real and sham stimulation effects on corticospinal excitability, as measured by change in $1 \mathrm{mV}$ MEP amplitude at various time points, in the real (blue line) and sham (red line) stimulation groups. Error bars represent standard errors. ${ }^{*} p<0.05$ versus real stimulation. ${ }^{\circ} p<0.05$ versus baseline (T0). MEP motor evoked potential, $m V$ millivolt, min minutes 
(at least 30\%), for even a longer time. As reported above, tissue biostimulation provided by CTU Meg 20 was based on non-ionizing LF-PEMFs, which could act primarily at synapse level, altering membrane ion channel function. In particular, it was shown that $\mathrm{Ca}^{2+}$ and $\mathrm{Na}^{+}$channel activity can be perturbed by magnetic fields, considering the diamagnetic anisotropic characteristics of membrane phospholipids [8, 30, 31]. Interestingly, CTU Mega 20 is defined as a diamagnetic acceleration system, allowing high-field magnetic impulses potentially capable of inducing magnetic reorientation of membrane phospholipids and consequently inducing a biological effect on nervous system function. From this point of view, there are three points in our proof of concept study that support the idea that non-ionizing LF-PEMFs induced by the CTU Mega 20 could represent a new approach for brain neuromodulation. They are (1) CTU Mega 20 provided a larger stimulation volume (up to $27 \mathrm{~cm}^{3}$ compared to $1-2 \mathrm{~cm}^{3}$ for TMS) allowing the modulation of an extended portion of cortical surface, compared to TMS; (2) as acommercially available device, the CTU Mega 20 is ready to use, with predefined programs to perform stimulation of the nervous system, not requiring specialized staff and can be easily adapted to everyday clinical application; (3) as a completely programmable system, the CTU Mega 20 can be optimized for different types of stimulation and different neurological diseases. In conclusion, the CTU Mega 20 diamagnetic acceleration system may well be of interest in the field of neuromodulation. Further studies to optimize protocol parameters for different neurological and psychiatric conditions are warranted [32, 33].

\section{Conclusions}

This proof-of-concept study in healthy subjects supports the idea that non-ionizing LF-PEMFs induced by the CTU Mega 20 diamagnetic acceleration system could represent a new approach for brain neuromodulation. Further studies to optimize protocol parameters for different neurological and psychiatric conditions are warranted.

\section{Additional files}

Additional file 1. CONSORT 2010 checklist. CONSORT 2010 checklist of information to include when reporting a randomised trial, with the number of the page for each item reported on the right.

Additional file 2. CTU Mega $20^{\circledR}$ technical description. Description: detailed description of the physical and technical basis of the functioning of CTU Mega $20^{\circledR}$ device used in the present study.
Hertz; eV: Electronvolt; CTU Mega 20: Name of the device used in the present study; TMS: Transcranial magnetic stimulation; MEP: Motor evoked potential; ms: Millisecond; min: Minute; J: Joule; $\mu$ s: Microsecond; FDI: First dorsal interosseous muscle; Ag/AgCl: Silver chloride; rMT: Resting motor threshold; EMG: Electromyography; $\mu \mathrm{V}$ : Microvolts; ANOVA: Analysis of variance.

\section{Authors' contributions}

All authors made substantial contributions to the study concept and design, and/or the acquisition of data, and/or the analysis and interpretation of the data. In detail, EP: first draft of the manuscript, patient cohort, interpretation of data, study conceptualization and design, AB: TMS data recording, TMS data analysis and interpretation of data, study conceptualization and design, critical revision of the manuscript for content, ALG: original developer of the (CTU) device used, technical description of the device, critical revision of the manuscript for content, SV, AC, NG: subject cohort, critical revision of the manuscript for content, VC: subject cohort, TMS data recording, AP: critical revision of the manuscript for content, BB, MM: study conceptualization and design, critical revision of the manuscript for content. All authors read and approved the final manuscript.

\section{Author details}

1 Stroke Unit, Azienda Socio Sanitaria Territoriale "Spedali Civili", "Spedali Civili" Hospital, Piazza Spedali Civili 1, 25123 Brescia, Italy. ${ }^{2}$ Neurology Unit, Department of Clinical and Experimental Sciences, University of Brescia, Brescia, Italy. ${ }^{3}$ cNVR Consorzio Veneto di Ricerca, Padua, Italy. ${ }^{4}$ Rehabilitation Unit, Casa di Cura "Villa Barbarano", Salò, Brescia, Italy.

\section{Acknowledgements}

We would like to thank all the partecipants (medical students, residents in Neurology, neuropsychologists) involved in the study.

\section{Competing interests}

This study was supported by PERISO SA (http://www.periso.ch/).

\section{Availability of data and materials}

Raw data are available to all interested researchers by direct request of the Corresponding Author (Dr. Premi, zedtower@gmail.com) or Dr. Magoni, (mauro.magoni@asst-spedalicivili.it) Head of the Stroke Unit, Azienda Socio Sanitaria Territoriale "Spedali Civili", "Spedali Civili" Hospital, Brescia, Italy.

\section{Consent for publication}

Written informed consent was obtained from study participants for participation in the study and for publication of this report and any accompanying images. Consent for publication was also obtained from the local ethics committee of our hospital in Brescia, Italy.

\section{Ethics approval and consent to participate}

All procedures performed in studies involving human participants were conducted in accordance with the 1964 Helsinki declaration as amended or comparable ethical standards and with the ethical standards of the Brescia Hospital (ASST Spedali Civili). Moreover, this study was approved (Authorization Number: 2322, March 09, 2016) by the ASST Spedali Civili Ethics Committee. Informed written consent to participate was obtained from each participant in the study.

\section{Funding}

This study was supported by PERISO SA (http://www.periso.ch/), which provided the CTU devices (real and sham) and the written technical description of the CTU device (see Additional file 2).

\section{Publisher's Note}

Springer Nature remains neutral with regard to jurisdictional claims in published maps and institutional affiliations.

Received: 10 November 2017 Accepted: 29 May 2018

Published online: 13 June 2018

\section{Abbreviations}

PAS: Paired associative stimulation; LTP: Long-term potentiation; LTD: Longterm depression; LF-PEMFs: Low frequency-pulsed electromagnetic fields; Hz: 


\section{References}

1. Stefan K, Kunesch E, Cohen LG, Benecke R, Classen J. Induction of plasticity in the human motor cortex by paired associative stimulation. Brain 2000;123(Pt 3):572-84

2. Huang YZ, Edwards MJ, Rounis E, Bhatia KP, Rothwell JC. Theta burst stimulation of the human motor cortex. Neuron. 2005;45(2):201-6.

3. Nitsche MA, Paulus W. Excitability changes induced in the human motor cortex by weak transcranial direct current stimulation. J Physiol. 2000:527(Pt 3):633-9.

4. Rossini PM, Burke D, Chen R, Cohen LG, Daskalakis Z, Di lorio R, Di Lazzaro V, Ferreri F, Fitzgerald PB, George MS, et al. Non-invasive electrical and magnetic stimulation of the brain, spinal cord, roots and peripheral nerves: basic principles and procedures for routine clinical and research application. An updated report from an I.F.C.N. Committee. Clin Neurophysiol. 2015;126(6):1071-107.

5. Benussi A, Cosseddu M, Filareto I, Dell'Era V, Archetti S, Sofia Cotelli M, Micheli A, Padovani A, Borroni B. Impaired long-term potentiation-like cortical plasticity in presymptomatic genetic frontotemporal dementia. Ann Neurol. 2016;80(3):472-6.

6. Di Lazzaro V, Oliviero A, Pilato F, Saturno E, Dileone M, Mazzone P, Insola A, Tonali PA, Rothwell JC. The physiological basis of transcranial motor cortex stimulation in conscious humans. Clin Neurophysiol. 2004;115(2):255-66

7. Hallett M. Transcranial magnetic stimulation and the human brain. Nature. 2000:406(6792):147-50.

8. Rosen AD. Mechanism of action of moderate-intensity static magnetic fields on biological systems. Cell Biochem Biophys. 2003;39(2):163-73.

9. Coots A, Shi R, Rosen AD. Effect of a 0.5-T static magnetic field on conduction in guinea pig spinal cord. J Neurol Sci. 2004;222(1-2):55-7.

10. Oliviero A, Mordillo-Mateos L, Arias P, Panyavin I, Foffani G, Aguilar J. Transcranial static magnetic field stimulation of the human motor cortex. J Physiol. 2011;589(Pt 20):4949-58.

11. Urnukhsaikhan E, Mishig-Ochir T, Kim SC, Park JK, Seo YK. Neuroprotective effect of low frequency-pulsed electromagnetic fields in ischemic stroke. Appl Biochem Biotechnol. 2017;181(4):1360-71.

12. Izzo MNL, Coscia V, La Gatta A, Mariani F, Gasbarro V. The role of diamagnetic pump (CTU Mega 18) in the physical treatment of limbs lymphoedema. A clinical study. Eur J Lymphol. 2010;21(61):24-9.

13. Juutilainen J. Developmental effects of electromagnetic fields. Bioelectromagnetics. 2005;Suppl 7:S107-115.

14. Seo TB, Kim TW, Shin MS, Ji ES, Cho HS, Lee JM, Kim TW, Kim CJ. Aerobic exercise alleviates ischemia-induced memory impairment by enhancing cell proliferation and suppressing neuronal apoptosis in hippocampus. Int Neurourol J. 2014;18(4):187-97.

15. Ceccarelli G, Bloise N, Mantelli M, Gastaldi G, Fassina L, De Angelis MG, Ferrari D, Imbriani M, Visai L. A comparative analysis of the in vitro effects of pulsed electromagnetic field treatment on osteogenic differentiation of two different mesenchymal cell lineages. Int Neurourol J. 2013:2(4):283-94

16. Maaroufi K, Save E, Poucet B, Sakly M, Abdelmelek H, Had-Aissouni L. Oxidative stress and prevention of the adaptive response to chronic iron overload in the brain of young adult rats exposed to a 150 kilohertz electromagnetic field. Neuroscience. 2011;186:39-47.

17. Levin M. Large-scale biophysics: ion flows and regeneration. Trends Cell Biol. 2007:17(6):261-70.
18. Pesce M, Patruno A, Speranza L, Reale M. Extremely low frequency electromagnetic field and wound healing: implication of cytokines as biological mediators. Eur Cytokine Netw. 2013;24(1):1-10.

19. Kang KS, Hong JM, Kang JA, Rhie JW, Jeong YH, Cho DW. Regulation of osteogenic differentiation of human adipose-derived stem cells by controlling electromagnetic field conditions. Exp Mol Med. 2013;45:e6.

20. Martiny K, Lunde M, Bech P. Transcranial low voltage pulsed electromagnetic fields in patients with treatment-resistant depression. Biol Psychiatry. 2010;68(2):163-9.

21. Rohan ML, Yamamoto RT, Ravichandran CT, Cayetano KR, Morales OG, Olson DP, Vitaliano G, Paul SM, Cohen BM. Rapid mood-elevating effects of low field magnetic stimulation in depression. Biol Psychiatry. 2014;76(3):186-93.

22. Straaso B, Lauritzen $L$, Lunde $M$, Vinberg $M$, Lindberg $L$, Larsen ER, Dissing $\mathrm{S}$, Bech P. Dose-remission of pulsating electromagnetic fields as augmentation in therapy-resistant depression: a randomized, double-blind controlled study. Acta Neuropsychiatr. 2014;26(5):272-9.

23. Volkow ND, Tomasi D, Wang GJ, Fowler JS, Telang F, Wang R, Alexoff D, Logan J, Wong C, Pradhan K, et al. Effects of low-field magnetic stimulation on brain glucose metabolism. Neuroimage. 2010;51(2):623-8.

24. Bassett CA. Fundamental and practical aspects of therapeutic uses of pulsed electromagnetic fields (PEMFs). Crit Rev Biomed Eng. 1989;17(5):451-529.

25. Luben RA. Effects of low-energy electromagnetic fields (pulsed and DC) on membrane signal transduction processes in biological systems. Health Phys. 1991;61(1):15-28.

26. Patino O, Grana D, Bolgiani A, Prezzavento G, Mino J, Merlo A, Benaim F. Pulsed electromagnetic fields in experimental cutaneous wound healing in rats. J Burn Care Rehabil. 1996;17(6 Pt 1):528-31.

27. Benussi A, Cotelli MS, Cosseddu M, Bertasi V, Turla M, Salsano E, Dardis A, Padovani A, Borroni B. Preliminary results on long-term potentiation-like cortical plasticity and cholinergic dysfunction after miglustat treatment in niemann-pick disease type C. In: JIMD Report 2017.

28. Fiorio M, Emadi Andani M, Marotta A, Classen J, Tinazzi M. Placeboinduced changes in excitatory and inhibitory corticospinal circuits during motor performance. J Neurosci. 2014;34(11):3993-4005.

29. Rivadulla C, Foffani G, Oliviero A. Magnetic field strength and reproducibility of neodymium magnets useful for transcranial static magnetic field stimulation of the human cortex. Neuromodulation. 2014;17(5):438-41

\section{(Discussion 441-432).}

30. Lu XW, Du L, Kou L, Song N, Zhang YJ, Wu MK, Shen JF. Effects of moderate static magnetic fields on the voltage-gated sodium and calcium channel currents in trigeminal ganglion neurons. Electromagn Biol Med. 2015;34(4):285-92.

31. Ye SR, Yang JW, Chen CM. Effect of static magnetic fields on the amplitude of action potential in the lateral giant neuron of crayfish. Int J Radiat Biol. 2004:80(10):699-708.

32. Rohan M, Parow A, Stoll AL, Demopulos C, Friedman S, Dager S, Hennen J, Cohen BM, Renshaw PF. Low-field magnetic stimulation in bipolar depression using an MRI-based stimulator. Am J Psychiatry. 2004;161(1):93-8.

33. Capone F, Dileone M, Profice P, Pilato F, Musumeci G, Minicuci G, Ranieri F, Cadossi R, Setti S, Tonali PA, et al. Does exposure to extremely low frequency magnetic fields produce functional changes in human brain? J Neural Transm (Vienna). 2009:116(3):257-65. 